\title{
BMJ Open Educational resource for antimicrobial resistance and stewardship for dentistry programmes: a research protocol
}

\author{
Chuan Yok Ang, ${ }^{1}$ Jagjit Singh Dhaliwal (D , ${ }^{1}$ Siti Hanna Muharram, \\ Muhammad Eid Akkawi (1) , 2 Zahid Hussain, ${ }^{3}$ Hanif Rahman, ${ }^{1}$ Yuh Yen Kok, ${ }^{1}$ \\ Sachinjeet Kaur Sodhi Dhaliwal, ${ }^{1}$ Long Chiau Ming (D) ${ }^{1}$
}

To cite: Ang CY, Dhaliwal JS, Muharram SH, et al. Educational resource for antimicrobial resistance and stewardship for dentistry programmes: a research protocol. BMJ Open 2021;11:e048609. doi:10.1136/ bmjopen-2021-048609

- Prepublication history and additional supplemental material for this paper are available online. To view these files, please visit the journal online (http://dx.doi.org/10.1136/ bmjopen-2021-048609).

Received 01 January 2021 Accepted 21 June 2021

Check for updates

(c) Author(s) (or their employer(s)) 2021. Re-use permitted under CC BY-NC. No commercial re-use. See rights and permissions. Published by BMJ.

${ }^{1}$ Pengiran Anak Puteri Rashidah Sa'adatul Bolkiah Institute of Health Sciences, Universiti Brunei Darussalam (UBD),

Gadong, Brunei Darussalam

${ }^{2}$ Kulliyyah of Pharmacy, International Islamic University, Kuantan Campus, Kuantan, Malaysia

${ }^{3}$ University of Canberra, Canberra, Australian Capital Territory, Australia

Correspondence to Dr Jagjit Singh Dhaliwal; jagjit.dhaliwal@ubd.edu.bn

\section{ABSTRACT}

Introduction Antimicrobial resistance (AMR) is a global public and patient safety issue. With the high AMR risk, ensuring that the next generation of dentists that have optimal knowledge and confidence in the area of AMR is crucial. A systematic approach is vital to design an AMR content that is comprehensive and clinically relevant. The primary objective of this research study will be to implement a consensus-based approach to elucidate AMR content and curriculum priorities for professional dentistry programmes. This research aims to establish consensus along with eliciting opinion on appropriate AMR topics to be covered in the Bachelor of Dental Surgery syllabus. Methods and analysis A three-phase approach to validate content for curriculum guidelines on AMR will be adopted. First, literature review and content analysis were conducted to find out the available pertinent literature in dentistry programmes. A total of 23 potential literature have been chosen for inclusion within this study following literature review and analysis in phase 1 . The materials found will be used to draft curriculum on antimicrobials for dentistry programmes. The next phase involves the validation of the drafted curriculum content by recruiting local and foreign experts via a survey questionnaire. Finally, Delphi technique will be conducted to obtain consensus on the important or controversial modifications to the revised curriculum.

Ethics and dissemination An ethics application is currently under review with the Institute of Health Science Research Ethics Committee, Universiti Brunei Darussalam. All participants are required to provide a written consent form. Findings will be used to identify significant knowledge gaps on AMR aspect in a way that results in lasting change in clinical practice. Moreover, AMR content priorities related to dentistry clinical practice will be determined in order to develop need-based educational resource on microbes, hygiene and prudent antimicrobial use for dentistry programmes.

\section{INTRODUCTION}

Antimicrobial resistance (AMR) poses a significant threat to the environment and public health globally. AMR occurs when microbes initially sensitive to an antimicrobial medicine becomes resistant. This major problem is aggravated by the scarcity of

\section{Strengths and limitations of this study}

- The Delphi method was widely used to research healthcare curriculum design and development, but this protocol signifies the first application in the antimicrobial resistance (AMR) field for professional undergraduate dental degree.

- Validated consensus method will be used to design an updated and relevant AMR curriculum for professional dental degree by an international expert panel.

- Top AMR content priorities related to dentistry clinical practice will be determined in order to develop need-based educational resource on microbes, hygiene and prudent antimicrobial use for dentistry programmes.

- The suggestions provided by Delphi panellists may not completely represent the whole spectrum of experts directly or indirectly involved in AMS education and AMR. Furthermore, real evidence on the usefulness of the newly custom-made AMR curriculum for dental programmes is still needed.

new and effective antimicrobials to combat resistant microbes. ${ }^{1}$ Direct implications to the widespread resistant strains of microbes include among others, prolonged hospitalisation, high mortality rate, high medical costs and in extreme cases, may lead to untreatable illnesses. ${ }^{2}$ Left unaddressed, deaths attributed to AMR are expected to rise up to 10 million every year by 2050 , surpassing cancer as the main causes of mortality. ${ }^{34}$

Antimicrobial stewardship (AMS), an integrated approach that involves optimal selection, dosing, route of administration and duration of antimicrobial treatment, is considered crucial in the treatment of patients in all fields and it plays a pivotal role in averting the occurrence of AMR. ${ }^{25}$ Numerous AMS education has sought to teach proper prescription habits to physicians with the aim to modify their prescribing behaviour ${ }^{5-7}$; however, achieving that has proven challenging. ${ }^{89}$ 
Despite a vast majority of the community taking up AMS education, there is still a paucity of evidence concerning broad engagement with AMS across medical subspecialties, which is essential as a significant proportion of antimicrobial prescribers are not adept at dealing with infection management. ${ }^{10-14}$ In the context of dental practice, several interventions comprising audit, issuing of guidelines, provision of education and feedback or a combination of all have resulted in a significant reduction in antibiotic prescribing to some extent; however, dissemination of guidelines alone did not have any effect on prescribing. ${ }^{15}$ Moreover, AMS is poorly reported and much less executed in the dental compared with the medical context which calls for an established need to integrate AMS interventions in oral healthcare; although various interventions worldwide have already been in the pipeline to enhance dental antibiotic prescribing practices. ${ }^{15}$

Antibiotics, a subset of antimicrobials, are commonly prescribed by dentists in dental practice either to prevent diseases caused by infection or to treat diseases of the oral cavity. ${ }^{16}$ As professionals that are directly involved in the prescription of antibiotics, it is imperative that dentists improve antibiotic prescribing practices in an effort to curb the increasing emergence of antibiotic resistance or AMR as a whole as well as other adverse effects of drug abuse. ${ }^{16}$ In addition, a recent study conducted in the USA showed that antibiotic adverse effects (AAE), for example, anaphylaxis and C. difficile infection, though rare, did occur in patients within 14 days of unnecessary dental prophylaxis. ${ }^{17}$ It is even more concerning considering the fact that dentists may not be conscious of AAEs as most adverse effects are commonly diagnosed in medical settings. ${ }^{17}$ Hence, the abovementioned further reinforce the point made that more effective AMR and AMS education must be implemented to address this alarming issue.

Infections are a common occurrence in the clinical setting and the majority of dentistry students will prescribe an antibiotic at some point when they begin clinical practice. There is a limited availability of information regarding the nature or effectiveness of AMR education among undergraduate and postgraduate students. ${ }^{9} 1819$ It remains largely unknown whether this intervention would have a significant impact on clinical practice $^{1819}$ and consequently reducing resistance rates. The development of new and effective antibiotics is not able to keep up with the increasingly alarming rate of drug-resistance bacteria which calls for a more strategic approach to curb hundreds of thousands of deaths annually attributed to AMR. ${ }^{20}$

Several studies have demonstrated that students perceived antimicrobial education to be lacking in their dentistry degree programmes ${ }^{2122}$ which suggests the need for a greater emphasis on these areas. Furthermore, a recent survey showed that most university students had good knowledge related to AMR and correctly identified infections that can be treated with antibiotics as outlined by the WHO; however, there remain misconceptions on the usage of antibiotics for viral infections. ${ }^{4}$ This implies a need to further enhance education on appropriate antimicrobial use among the student population.

\section{OBJECTIVES}

The primary objective of this research study will be to implement a consensus-based approach to elucidate AMR content and curriculum priorities for professional dentistry programmes. The aim of this project is to develop need-based educational resource that is, a curriculum on microbes, hygiene and prudent antimicrobial use for dentistry programmes. This could provide a foundation of knowledge that will enhance understanding in antimicrobial use and promote the judicious use of antimicrobials.

\section{MATERIALS AND METHODS \\ Study design}

This study will involve a three-phase approach to validating content for curriculum guidelines on AMR and AMS. The study design was a modification of the approach implemented by Cumyn and Harris ${ }^{23}$ :

1. Literature review and qualitative content analysis to produce an initial draft curriculum on antimicrobials.

2. Validation of curriculum content by an expert panel consisting of both quantitative and qualitative survey methodologies.

3. Implementation of Delphi technique to obtain consensus on the more important or controversial modifications to the revised curriculum content.

\section{Phase 1: literature review and qualitative content analysis}

Literature review and qualitative content analysis from various online sources will be conducted to produce an initial draft curriculum on antimicrobials. Such sources are derived mainly from published syllabi or national guidelines.

\section{Data sources and search plan}

Electronic literature from various databases which met the inclusion criteria were identified and extracted for analysis. Such databases were PubMed, SCOPUS, Web of Science (WOS) and EBSCOhost (Medline Complete) which were used to search and browse all the literature (table 1); those literature possessing elements of a dental curriculum with relevance to antimicrobials or related matters were specially chosen. Other data sources included the employment of manual searching using Google Advanced Search and through reference lists of included literature to identify further literature. Additionally, if there is a need to search for a syllabus from specific universities or just as a general search, Google Advanced Search may serve as a supplementary manual search tool.

A search strategy was devised by the researcher (LCM) with four databases selected having comprehensive search 
Table 1 Summary of selected literatures detailing the search terms used, the search engine (PubMed, SCOPUS, WOS, EBSCOhost), the number of web results generated and the number selected from the results

\begin{tabular}{|c|c|c|c|}
\hline Search terms & Search engine & Results & Selected \\
\hline $\begin{array}{l}\text { (dentistry OR dental OR BDS OR Bachelor of Dental Surgery) AND (syllabus } \\
\text { OR curriculum OR learning objective OR learning aim) }\end{array}$ & PubMed & 429 & 0 \\
\hline $\begin{array}{l}\text { TITLE-ABS-KEY((dentistry OR dental OR ‘Bachelor Dental Surgery’) AND } \\
\text { (syllabus OR curriculum OR ‘learning objective’) AND (Anti-Infective OR } \\
\text { Anti Infective OR Antiinfective OR Microbicides OR Antimicrobial OR Anti- } \\
\text { Microbial OR Anti Microbial)) }\end{array}$ & SCOPUS & 3 & 1 \\
\hline $\begin{array}{l}\text { (dentistry OR dental OR ‘Bachelor Dental Surgery’) AND (syllabus OR } \\
\text { curriculum OR 'learning objective’) AND (Anti-Infective OR Anti Infective OR } \\
\text { Antiinfective OR Microbicides OR Antimicrobial OR Anti-Microbial OR Anti } \\
\text { Microbial) }\end{array}$ & $\begin{array}{l}\text { EBSCOhost } \\
\text { (Medline } \\
\text { Complete) }\end{array}$ & 322 & 3 \\
\hline \multicolumn{4}{|c|}{$\begin{array}{l}\text { N.B. } \\
\text { Total selected: } 10 \\
\text { Total duplicates: } 6 \text { (cross-checked with sources from each database and reference lists) } \\
\text { Final total: } 4\end{array}$} \\
\hline
\end{tabular}

terms in order to not omit any relevant potential primary literature, and the search itself was performed by the lead researcher (CYA). We centred our search primarily on the targeted programme's curriculum (dentistry in this case) and subsequently combined it with our research theme 'antimicrobials' to elicit the full potential of generating closely associated and appropriate literature by the databases. Additionally, attempts to narrow down the results generated from these selected databases were performed on the term 'antimicrobials' using MeSH (Medical Subject Headings) database due to the term's broad category; time constraints and to ensure high sensitivity were contributing factors for this search strategy.

\section{Inclusion and exclusion criteria}

\section{Criteria for studies to be included for reviewal}

In general, we included literature from any country and in the English language only. Literature are eligible for inclusion for reviewal irrespective of the publication date or study design. Literature containing all aspects relating to antimicrobials and AMR (not restricted to only antibacterial resistance) are included. AMS, an essential part of AMR aspect is to be included as well.

\section{Criteria for studies to be excluded}

Non-English and non-antimicrobials related literature are excluded. Any literature reporting knowledge, attitudes, practices or similar relating to antimicrobials without an educational intervention pertaining to published syllabi/curricula are to be excluded as well. Literature depicting any form of training of allied dental care professionals (dental nurses, dental hygienists, dental therapists, orthodontic therapists, dental technicians and clinical dental technicians) are not accepted for review.

\section{Search terms}

Several variants of terms and synonyms were employed for the purpose of avoiding failure of the database system to map to the most suitable term, and lexical ambiguity. The words "dentistry", "dental" and "Bachelor Dental Surgery"; "syllabus", "curriculum" and "learning objective"; "Anti-infective/Anti Infective/Antiinfective", "Microbicides" and "Antimicrobial/Anti-Microbial/Anti Microbial" were used interchangeably. However, for the purpose of simplicity and to save time, these terms were combined into one common search term: (dentistry OR dental OR "Bachelor Dental Surgery") AND (syllabus OR curriculum OR "learning objective") AND (Anti-Infective OR Anti Infective OR Antiinfective OR Microbicides OR Antimicrobial OR Anti-Microbial OR Anti Microbial) as shown in table 1 . 


\section{Selection procedure}

The titles of all literature were first read and assessed, and a significant number immediately found to be irrelevant. Titles which deemed suitable and relevant, abstracts were reviewed by the lead researcher. If found pertinent, full-text literature would then be assessed for eligibility and subsequently be included for further reviewal by other authors. The reference lists of included literature were screened to identify any additional relevant literature. This search strategy was applied across all databases chosen.

As many individual course syllabi are not usually published widely outside of a university, the literature obtained from the databases comprised any form of educational content related to AMR-competencies, learning outcomes or learning points along with their associated domains. Published syllabi, curricula or national guidelines were mainly derived using the Google Advanced Search tool. Following literature review and analysis in phase 1, a total of 23 articles have been chosen for inclusion within this study. Details of literatures/syllabi derived from each source-databases, Google Advanced Search and reference lists respectively were also presented (table 2).

\section{Evaluation of curriculum content}

A broad-based search criterion (online supplemental information 1) will be used to identify all syllabus (1) topics, and (2) learning points, which met the electronic search criteria. ${ }^{16}$ This curriculum search criteria (online supplemental information 1) will also help to assess the suitability and comprehensiveness of each syllabus obtained. The following curriculum information will be recorded: the year of publication, the total number of curriculum topics and individual learning points within. In the analysis, apart from AMS, we also intend to include individual learning points relating to AMR which include but not limited to antifungal, antibacterial, antiviral or antiprotozoal resistance; despite it has been widely known that most AMR are attributed to bacteria compared with other micro-organisms.

Following the identification of learning points for inclusion, variation between literature will be assessed via two methods. First, the proportions of AMR learning points will be calculated for each literature using the total number of learning points as a denominator. Second, the frequency of individual learning points per literature will be categorised according to the determined level of achievement that each individual learning point is expected to display. Adaptation of Miller's pyramid to rate the level of perceived output for individual learning points (level 1-4), along with examples of learning points that are categorised at each level will be used. The rating of learning points will have and is set to be done by thorough review and discussion among the research team.

Online supplemental information 2 illustrates a sample framework which will contain information on the topic and learning point selection process. ${ }^{16}$
Assessment of quality of included studies

Ultimately, all syllabi or curricula selected will be reviewed by the researchers to assess for appropriateness, comprehensiveness, clarity and accuracy of the content as well as if there is a need to include/modify/delete the content. Only content judged to be essential by the researchers will be included. Once finalised and consensus has been reached between the researchers, we will produce an initial draft curriculum on antimicrobials and proceed to phase 2 to validate the content.

\section{Phase 2: validation of the curriculum content}

Phase 2 involves the validation of the initial draft curriculum produced from phase 1 by a large consensus group of experts. Both quantitative and qualitative methodologies will be used. A curriculum consensus group consisting of local and foreign experts on the field of antimicrobials and related matters will be formed. This phase is further divided into two steps.

\section{Step 1: quantitative survey}

Experts will be asked to fill out an online questionnaire containing various topics on antimicrobial aspects coupled with their individual learning points. They will be asked to indicate for each item, whether to include/ modify/delete any items in the content as they see fit and subsequently provide justifications wherever applicable for each indication they make if necessary (figure 1). Suggestions to include any additional items can be made in the comment section provided at the end of every topic.

\section{Step 2: qualitative survey}

An interview-based survey where these experts will be invited to an online discussion to obtain their interpretation and feedback on the survey. This acts to reinforce step 1 and address any queries or doubts by the researchers. The results from the qualitative survey will be analysed and changes to be made to the updated draft curriculum from step 1; and phase 3 follows to obtain consensus on changes that could be made in the modified curriculum.

\section{Phase 3: implementation of Delphi technique}

The final phase involves the usage of Delphi technique to obtain consensus on the more important or controversial modifications to the revised curriculum in phase 2. Delphi technique uses a series of data collection rounds (usually 2 or 3 ) to garner views and judgments of a panel of experts on the topic of interest and achieve group consensus. This technique was selected to ensure that no potential areas of the curriculum are omitted. One benefit of this technique is that it allows for subjective input from a larger group of participants with a wide array of expertise to obtain consensus. $^{23}$

As opposed to phase 2, this may involve a different group of experts. It is noteworthy that the decided number of rounds for Delphi survey is two rather than being determined by the number needed to achieve 
Table 2 Details of potential literatures/syllabi from various sources

Title

Setting

Year published

(A) Databases (PubMed, SCOPUS, WOS and EBSCO) after duplicates removed ( $n=4)$

Role of Education in AMS

The Netherlands 2018

AMR and global public health crisis: are we educating enough our future prescribers to Malaysia 2020 combat this global threat?

Raising awareness about microbial antibiotic resistance in undergraduate dental $\quad$ Spain 2020 students: a research-based strategy for teaching non-laboratory elements of a microbiology curriculum

Saudi Board in Restorative Dentistry (SBRD) curriculum using CanMEDS competency Saudi Arabia 2018

(B) Manual search (Google Advanced Search) ( $n=12)$

NHS Embedding national antimicrobial prescribing and stewardship competencies into UK

2016

curricula

Committee of Postgraduate Dental Deans and Directors UK (COPDEND) Dental $\quad$ UK 2015

Foundation Training Curriculum 2015

Dental Council of India Syllabus for Undergraduate (BDS) India Not stated

Ghana National Action Plan on AMR 2017-2021

Ghana, West 2017

Africa

The Bachelor of Dental Surgery Curriculum B.P. Koirala Institute of Health Sciences $\quad$ Nepal 1999 Dharan, Nepal

Syllabus and Curriculum BDS Degree Course Jamia Millia Islamia, New Delhi India Not stated

B.D.S Course Regulations/Syllabus Kerala University of Health Sciences $\quad$ India 2010-2011

HKU Faculty of Dentistry 5-Year BDS Curriculum Handbook

Hong Kong 2014

National University of Medical Sciences 2nd Year BDS Curriculum (Revised 2018) $\quad$ Pakistan 2018

Dental Curriculum Bangladesh

Bangladesh 2002-2003 (not stated clearly)

Implementation of an Outcome-Based Longitudinal Pharmacology Teaching in $\quad$ Saudi Arabia 2017

Undergraduate Dental Curriculum at KSAU-HS Experience

Health Workers' Education And Training On AMR: Curricula Guide $\quad$ UK 2019

(C) Reference lists ( $n=7$ )

Mapping AMS in undergraduate medical, dental, pharmacy, nursing and veterinary

UK

2016

education in the United Kingdom

\begin{tabular}{lll} 
Educational effectiveness, target, and content for prudent antibiotic use & Korea & 2015 \\
\hline Undergraduate curriculum guidelines for endodontology & UK & 2013 \\
$\begin{array}{l}\text { How to educate prescribers in AMS practices } \\
\begin{array}{l}\text { Development of an educational resource on microbes, hygiene and prudent antibiotic } \\
\text { use for junior and senior school children }\end{array}\end{array}$ & France & 2013 \\
$\begin{array}{l}\text { Overview of e-Bug: An antibiotic and hygiene educational resource for schools } \\
\begin{array}{l}\text { Evaluation of e-Bug, an educational pack, teaching about prudent antibiotic use and } \\
\text { hygiene, in the Czech Republic, France and England }\end{array}\end{array}$ & UK & 2011 \\
\hline
\end{tabular}

hygiene, in the Czech Republic, France and England

a predetermined level of consensus in this study. The underlying reasons behind this decision are to avoid burdening participants with lengthy surveys and the likelihood of participants reaching an artificial consensus for the sake of completing the job as well as the possibility of participant fatigue.

In contrast to phase 2, a different approach to the analysis of data for both rounds in this phase will be conducted by the researchers. To our knowledge, there are no internationally recognised criterion standards to what constitutes a consensus. Any items achieving a consensus level of $100 \%$ will be accepted outright. Only items achieving at least $81 \%$ (between $80 \%$ and $100 \%$ ) agreement will be accepted but modified if there are suggestions up to the level of agreement. Additionally, items with less than or equal to $80 \%$ consensus level (based on researchers' decision) will be rejected unless modifications or comments are made in the space provided on how these could be improved. In this phase, a two-round Delphi survey will be adopted. 


\begin{tabular}{|c|c|c|c|}
\hline \multicolumn{4}{|c|}{$\begin{array}{l}\text { Please indicate with }(\checkmark) \text { for each item: include / modify / delete. Suggestion(s) to include any additional items can } \\
\text { be made in the comment section at the end of every topic. }\end{array}$} \\
\hline Topic 1: Infection Prevention and Control & Include & Modify & Delete \\
\hline \multicolumn{4}{|l|}{$\begin{array}{l}\text { Learning Point 1: Understand the role of hand hygiene to prevent } \\
\text { transmission of pathogens }\end{array}$} \\
\hline \multicolumn{4}{|l|}{ Justification: } \\
\hline \multicolumn{4}{|l|}{$\begin{array}{l}\text { Learning Point 2: Promote principles of healthcare-associated } \\
\text { infections (HAI) prevention and control }\end{array}$} \\
\hline Justification: & & & \\
\hline
\end{tabular}

Figure 1 Example of survey format to be employed.

\section{Round 1 survey}

The first round of the Delphi survey will contain the updated content from phase 2. Delphi participants will be asked to use a five-point Likert scale (1=strongly disagree; $2=$ disagree; $3=$ neutral; $4=$ agree and $5=$ strongly agree) to rate their level of agreement with the proposed modifications (figure 2). A space in the survey will also be provided to invite participants to provide a brief justification or reference to support their rating choice based on the suggested additions/modifications derived from phase 2 and their rationale.

\section{Round 2 survey}

The second-round survey will be administered in a similar manner as round 1. However, only Delphi participants who managed to complete round 1 will be invited to proceed to round 2. Prior to the commencement of this round, participants will be presented the quantitative results from round 1 to invite their interpretation and feedback; this may be done qualitatively to derive a more comprehensive response. In the case of any items for which there is a lack of agreement, items which are modified after taking into account the qualitative feedback, and additional items identified by the participants, they will be included in the second-round survey. Following the completion of this round, an analysis will be done by the researchers alone to review and obtain final consensus on the improved curriculum. Correction of any typographical errors made by the Delphi participants, refining and standardisation of formatting will also be done in this final step.

\section{Recruitment and identification of experts}

Experts will be recruited as panel members based on their knowledge, skills and experiences. The aim is to recruit up to 200 participants (dentists/dental specialists;

\begin{tabular}{|l|l|}
\hline Background Information: \\
Here is a list of items as suggested by some respondents. We wish to remind you that the aim of the \\
research is to develop a syllabus on antimicrobials for Dentistry programmes; this could provide a \\
foundation of knowledge that will enhance understanding in antimicrobial use and promote the judicious \\
use of antimicrobials in clinical practice. For each item, please rate the extent to which you agree or \\
disagree using the scale below.
\end{tabular}

Figure 2 Example of Delphi survey format to be employed. 
infectious disease specialists; infection control practitioners; physicians; microbiologists or similar) in both the quantitative part of the study and the qualitative part of the study, as suggested by extant literature. Since there is no established definition to what constitutes an 'expert', we have assumed the definition, 'individuals reflecting expertise in prescribing and medicines management with regards to the education and practice of dentist and dental surgeon; and antimicrobial prescribing and stewardship'. ${ }^{2}$ To our knowledge, there is no firm consensus on the number of experts required for this study as sample size would depend largely on the purpose of the research, the complexity of the problem to be investigated and availability of resources. ${ }^{2}$

Expert panel members mainly consisting of qualified dentistry academicians and dental education professionals will be identified and recruited from various countries (including Brunei Darussalam, Malaysia, Australia and Indonesia) using the purposive and snowball sampling methods. In the purposive method, qualified participants who meet the 'expert' criteria will be identified and approached by the researchers. Using the snowball sampling method, identified participants may then refer researchers to other participants who are qualified. All participants will each be allocated an identification number for easy collection and reporting of the results. Every panel member will be sent a Participant Information Sheet with all the necessary information about the study via email and should they have any queries, it will be addressed by a researcher. A consent form will also need to be signed and dated prior to completing the questionnaire.

\section{Data collection and data analysis}

The study is expected to commence in December 2020 and to finish in December 2021. There will be a total of three rounds to the quantitative survey; one survey from phase 2 and two from phase 3. In order that data collection from a large number of participants be conducted timely and efficiently, Qualtrics, a commonly used web survey tool in the university, will be used to develop each round of the online survey. Data will be collected on Qualtrics and the survey link will be disseminated to an identified representative from each institution to be distributed to all participants that are eligible via email. Once participation has been confirmed, participants will be sent an email containing invitation links to the online surveys on specified dates. Follow-up reminder emails will be forwarded to the participants to encourage responsiveness.

All completed surveys will be analysed by the principal investigator. Data analysis will be done using RStudio statistical software for Windows. Inferential and descriptive statistical methods will be used. Responses of qualitative nature (justifications/suggestions) will be coded to identify different themes and discussions to be held among the research team if any disparities or doubts arise to achieve consensus.

\section{Ethical consideration}

The study will be conducted in accordance with principles of the Good Clinical Practice and Declaration of Helsinki. Ethics approval has been sought from the Institute of Health Science Research Ethics Committee (IHSREC), Universiti Brunei Darussalam to conduct the study. No personal identifying information will be collected so the participants will be anonymous when the questionnaires are filled and collected. However, other demographic information of the participants such as their principal role, age, qualifications, place of practice and contact details may be collected; this information is recorded in order to report the range of expertise within the panel. Participants will also be asked to provide their name and consent to be acknowledged and included as a member of the expert panel in publications of this study. Additionally, participants are free to withdraw at any time by sending an email to the researcher informing their participant code and their decision to withdraw from the study.

\section{Patient and public involvement}

Patients or the public were not involved in the design, or conduct, or reporting, or dissemination plans of our research.

\section{DISCUSSION}

Undeniably, knowledge gaps around AMR still exist in every part of the world. To address this, a comprehensive research strategy and evaluation involving a collaboration of various stakeholders and by actively conducting clinical trials would be necessary to mitigate the upsurge of resistance.

To the best of our knowledge, there has never been any formalised study conducted to develop a curriculum on AMR and antimicrobials tailored to dentistry programmes in the country. Likewise, there is no documented information investigating dentistry students' perceptions of the need for antimicrobial education or the extent of their preparedness to appropriately prescribe antibiotics. At present, the content used for AMR is not validated. In this study, a comprehensive and updated curriculum will be developed which will then be assimilated into the existing dentistry programmes.

Through better understanding the spectrum of various curriculum on AMR principles and practices and how these are being assessed, we may be able to use the findings to adopt suitable teaching methodologies that effectively educate students. Moreover, such findings may be invaluable in helping to identify significant gaps surrounding our knowledge, attitudes and awareness related to antimicrobials in a way that results in lasting change in the clinical environment.

\section{Expected limitation}

The panel of experts will be approached through sampling and its composition may not cover the whole spectrum of professions involved in AMR and its related 
aspects, for example, infectious disease specialists and microbiologists.

Acknowledgements Authors would like to thank Mr Awangku Muhammad Adi Marhain Bin Pengiran Haji Mohamad Yusra for his support in ethical application documentation.

Contributors LCM conceived the conceptual framework and designed the search strategy. CYA wrote and developed the study protocol. LCM, JSD, SHM, MEA, SKSD, YYK, HR and ZH revised and reviewed the final protocol. All authors read and approved the final version.

Funding This study was funded by Universiti Brunei Darussalam, publication fee grant.

Competing interests None declared.

Patient consent for publication Not required.

Provenance and peer review Not commissioned; externally peer reviewed.

Supplemental material This content has been supplied by the author(s). It has not been vetted by BMJ Publishing Group Limited (BMJ) and may not have been peer-reviewed. Any opinions or recommendations discussed are solely those of the author(s) and are not endorsed by BMJ. BMJ disclaims all liability and responsibility arising from any reliance placed on the content. Where the content includes any translated material, BMJ does not warrant the accuracy and reliability of the translations (including but not limited to local regulations, clinical guidelines, terminology, drug names and drug dosages), and is not responsible for any error and/or omissions arising from translation and adaptation or otherwise.

Open access This is an open access article distributed in accordance with the Creative Commons Attribution Non Commercial (CC BY-NC 4.0) license, which permits others to distribute, remix, adapt, build upon this work non-commercially, and license their derivative works on different terms, provided the original work is properly cited, appropriate credit is given, any changes made indicated, and the use is non-commercial. See: http://creativecommons.org/licenses/by-nc/4.0/.

\section{ORCID iDs}

Jagjit Singh Dhaliwal http://orcid.org/0000-0002-3357-388X

Muhammad Eid Akkawi http://orcid.org/0000-0001-9964-0355

Long Chiau Ming http://orcid.org/0000-0002-6971-1383

\section{REFERENCES}

$1 \mathrm{Hu} X-Y$, Logue M, Robinson N. Antimicrobial resistance is a global problem - a UK perspective. Eur J Integr Med 2020;36:101136.

2 Courtenay M, Lim R, Castro-Sanchez E, et al. Development of consensus-based national antimicrobial stewardship competencies for UK undergraduate healthcare professional education. J Hosp Infect 2018;100:245-56.

3 Venter $\mathrm{H}$, Henningsen ML, Begg SL. Antimicrobial resistance in healthcare, agriculture and the environment: the biochemistry behind the headlines. Essays Biochem 2017;61:1-10.

4 Shahpawee NS, Chaw LL, Muharram SH, et al. University students' antibiotic use and knowledge of antimicrobial resistance: what are the common myths? Antibiotics 2020;9. doi:10.3390/ antibiotics9060349. [Epub ahead of print: 20 Jun 2020].
5 Silverberg SL, Zannella VE, Countryman D, et al. A review of antimicrobial stewardship training in medical education. Int $\mathrm{J}$ Med Educ 2017;8:353-74.

6 Dellit TH, Owens RC, McGowan JE, et al. Infectious diseases Society of America and the Society for healthcare epidemiology of America guidelines for developing an institutional program to enhance antimicrobial stewardship. Clin Infect Dis 2007;44:159-77.

7 Roumie CL, Halasa NB, Edwards KM, et al. Differences in antibiotic prescribing among physicians, residents, and nonphysician clinicians. Am J Med 2005;118:641-8.

8 Davey Pet al. Interventions to improve antibiotic prescribing practices for hospital inpatients. Cochrane Database Syst Rev 2005;4:Cd003543. doi:10.1002/14651858.CD003543.pub2

9 Pulcini C, Wencker F, Frimodt-Møller N, et al. European survey on principles of prudent antibiotic prescribing teaching in undergraduate students. Clin Microbiol Infect 2015;21:354-61.

10 Pulcini C, Williams F, Molinari N, et al. Junior doctors' knowledge and perceptions of antibiotic resistance and prescribing: a survey in France and Scotland. Clin Microbiol Infect 2011;17:80-7.

11 Howard P, Pulcini C, Levy Hara G, et al. An international crosssectional survey of antimicrobial stewardship programmes in hospitals. J Antimicrob Chemother 2015;70:1245-55.

12 Pulcini C, Cua E, Lieutier F, et al. Antibiotic misuse: a prospective clinical audit in a French university hospital. Eur J Clin Microbiol Infect Dis 2007;26): :277-80.

13 Zarb P, Coignard B, Griskeviciene J, et al. The European Centre for Disease Prevention and Control (ECDC) pilot point prevalence survey of healthcare-associated infections and antimicrobial use. Euro Surveill 2012;17. doi:10.2807/ese.17.46.20316-en. [Epub ahead of print: 15 Nov 2012].

14 Rawson TM, Moore LSP, Gilchrist MJ, et al. Antimicrobial stewardship: are we failing in cross-specialty clinical engagement? $J$ Antimicrob Chemother 2016;71:554-9.

15 Teoh L, Thompson W, Suda K. Antimicrobial stewardship in dental practice. J Am Dent Assoc 2020;151:589-95.

16 Oberoi SS, Dhingra C, Sharma G, et al. Antibiotics in dental practice: how justified are we. Int Dent J 2015;65:4-10.

17 Gross AE, Suda KJ, Zhou J, et al. Serious antibiotic-related adverse effects following unnecessary dental prophylaxis in the United States. Infect Control Hosp Epidemiol 2021;42:110-2.

18 Castro-Sánchez E, Drumright LN, Gharbi M, et al. Mapping antimicrobial stewardship in undergraduate medical, dental, pharmacy, nursing and veterinary education in the United Kingdom. PLoS One 2016;11:e0150056.

19 Rawson TM, Butters TP, Moore LSP, et al. Exploring the coverage of antimicrobial stewardship across UK clinical postgraduate training curricula. J Antimicrob Chemother 2016;71:3284-92.

20 Lomazzi M, Moore M, Johnson A, et al. Antimicrobial resistance moving forward? BMC Public Health 2019;19:858.

21 Dyar OJ, Howard P, Nathwani D, et al. Knowledge, attitudes, and beliefs of French medical students about antibiotic prescribing and resistance. Med Mal Infect 2013;43:423-30.

22 Abbo LM, Cosgrove SE, Pottinger PS, et al. Medical students perceptions and knowledge about antimicrobial stewardship: how are we educating our future prescribers? Clin Infect Dis 2013;57:631-8.

23 Cumyn A, Harris IB. A comprehensive process of content validation of curriculum consensus guidelines for a medical specialty. Med Teach 2012;34:e566-72. 